\title{
Fertility preservation in women with cancer
}

\author{
Sanghoon Lee', Jae Yun Song', Seung Yup Ku², Sun Haeng Kim', Tak Kim \\ ${ }^{1}$ Department of Obstetrics and Gynecology, Korea University College of Medicine; ${ }^{2}$ Department of Obstetrics and Gynecology, Seoul National \\ University College of Medicine, Seoul, Korea
}

\begin{abstract}
Fertility preservation (FP) is an effort to retain the fertility of cancer patients, and as an emerging discipline, it plays a central role in cancer care. Because of improvement in diagnostic and therapeutic strategies, an increasingly large number of patients are surviving with cancer. FP specialists should make an effort to spread the significance of FP among reproductive women with cancer and provide appropriate education both for associated physicians and for cancer patients who wish to preserve their fertility. Physicians who take part in the initial diagnosis and management of cancer should consider the importance of early referral of young cancer patients to FP specialists and take care of those patients by providing timely information and appropriate counseling. Individualized treatment strategies should be delivered depending on the patient's situation with appropriate team approach.
\end{abstract}

Keywords: Fertility preservation; Cancer; Referral; Women; Physicians

\section{Introduction}

Fertility preservation (FP) is an effort to help cancer patients retain their fertility and an emerging discipline that now plays a central role in the care of reproductive women with cancer. Because of improvement in diagnostic and therapeutic strategies, an increasingly large number of patients are surviving with cancer. For example, the five year-survival rate of breast cancer including all cancer stages has reached $89 \%$ [1]. The number of young cancer survivors among women diagnosed with cancer has been continuously increasing [2]. As a result, quality-of-life issues, including future pregnancies after cancer treatment, have gained significant importance in cancer care.

\section{Gonadal damage}

Chemotherapy and radiotherapy can cause severe gonadal damage resulting in amenorrhea due to ovarian follicle loss in females.

Received: Jun 15, 2012 · Accepted: Jun 21, 2012

Corresponding author: Tak Kim

Department of Obstetrics and Gynecology, Korea University College of

Medicine, 73 Inchon-ro, Seongbuk-gu, Seoul 136-705, Korea

Tel: +82-2-920-6842 Fax:+82-2-921-5357 E-mail: tkim@kumc.or.kr

This is an Open Access article distributed under the terms of the Creative Commons Attribution Non-Commercial License (http://creativecommons.org/licenses/by-nc/3.0/) which permits unrestricted non-commercial use, distribution, and reproduction in any medium, provided the original work is properly cited.
Adjuvant chemotherapy, particularly with alkylating agents such as cyclophosphamide, is gonadotoxic and induces premature ovarian failure. The drugs are generally classified as high risk (e.g., cyclophosphamide, chlorambucil, melphalan, busulfan, nitrogen mustard, and procarbazine), intermediate risk (e.g., cisplatin, and adriamycin), and low risk (e.g., methotrexate, 5-fluorouracil [CMF], vincristine, bleomycin, and actinomycin D) [3] (Table 1). The degree of chemotherapyinduced ovarian damage is dependent on the patient's age, the drug used, and the dosage of the drugs. Since most cancer patients are treated with multi-agent chemotherapy protocols, it is not easy to assess the degree of gonadal damage. Radiotherapy-induced follicular damage resulting in a high risk of prolonged amenorrhea in women can occur when women are exposed by pelvic or whole abdominal radiation dose $\geq 6 \mathrm{~Gy}$ in adult women, $\geq 10 \mathrm{~Gy}$ in postpubertal girls, and $\geq 15$ Gy in prepubertal girls [4-8]. The radiation dose of concurrent chemoradiation therapy (CCRT) for patients with advanced stage cervical cancer is usually about $50 \mathrm{~Gy}$. Thus gynecologic oncologists should consider the possibility of infertility in patients who undergo CCRT.

\section{Candidates and determinants of access to fertility preservation}

Candidates for FP include patients with childhood cancers, breast 
Table 1. The degree of gonadal damage by chemotherapy

\begin{tabular}{lcl}
\hline High risk & Intermediate risk & \multicolumn{1}{c}{ Low risk } \\
\hline Cyclophosphamide & Cisplatin & Methotrexate \\
Chlorambucil & Adriamycin & 5-Fluorouracil \\
Melphalan & & Vincristine \\
Busulfan & & Bleomycin \\
Nitrogen Mustard & & Actinomycin D \\
Procarbazine & \\
\hline
\end{tabular}

cancer, gynecologic cancers, hematologic cancers such as leukemia and lymphoma, those who need hematopoietic stem cell transplantation or pelvic irradiation for other diseases, and those with a high risk of premature ovarian failure (also called primary ovarian insufficiency). Since numerous departments are involved in FP treatment, collaborating as a team with several specialists who take part in the initial cancer diagnosis and treatment, including a mental health provider, is necessary to manage cancer patients.

Although under ideal circumstances patients should be referred to FP specialists before chemotherapy, many of those who did not have this opportunity may develop infertility and are referred for post-chemotherapy assisted reproduction [9]. Because the likelihood of ART success post-chemotherapy is significantly diminished [10], it is extremely important to understand the factors that determine the access to FP and early referral. In a previous study, we evaluated the socioeconomic, demographic, and medical factors that influence early referral before cancer treatment to FP versus delayed referral to postchemotherapy assisted reproduction in 314 women with breast cancer. Factors favoring referrals for FP were older age, early stage breast cancer, receiving cancer care at an academic center, and family history of breast cancer [11]. This information has revealed the barriers to accessing early FP care.

\section{Importance of early referral to fertility preservation}

Oncologists especially should recognize the importance of FP and early referral to specialists. Both embryo and oocyte freezing for FP require ovarian stimulation with gonadotropins. Since ovarian stimulation must be started within the first four days of the menstrual cycle to be effective, and requires approximately two weeks for completion, early referral is crucial to avoid a delay in chemotherapy. The author's previous study concluded that early referral prior to breast surgery enables women with breast cancer to initiate an FP cycle sooner and undergo multiple cycles of oocyte or embryo freezing, if desired. Referral prior to breast surgery $(n=35)$ results in the initiation of chemotherapy on average 3 weeks earlier in single FP cycles, as well as when the data from double cycles are included, compared to those referred after breast surgery $(n=58)$. This additional time enables multiple cycles of FP ( $9 / 35$ vs. 1/58). Women who can undergo multiple cycles are likely to be at an advantage for FP because of the additional number of eggs or embryos generated [12].

A recent study, however, indicated that still less than half of physicians routinely refer cancer patients of childbearing age to reproductive specialists [13]. Another recent study reported that while most oncologists recognize the importance of discussing infertility risks after cancer treatment, few actually discuss FP with their patients [14]. In addition, Armuand et al. [15] reported that there have been sex differences in access to fertility-related information and the use of FP treatment. Only half of the women had discussed fertility issues with a health care professional, although the majority of men had received information about FP such as sperm cryopreservation [15]. Potential explanations are that sperm banking for men is an easy and well established method, and the delay is negligible compared to the required duration of ovarian stimulation for embryo or oocyte cryopreservation.

Oncologists play a key role in understanding patients' concerns regarding fertility. FP specialists should make an effort to publicize the significance of FP for reproductive women with cancer and should provide appropriate education both for associated physicians and for cancer patients who wish to preserve their fertility. As important as it is to encourage oncologists to refer young people with cancer to FP counseling, it is just as important to emphasize referral as early in the process as possible to maximize the likelihood of success.

\section{Standard methods for fertility preservation}

The American Society of Clinical Oncology (ASCO) has issued practice guidelines for FP options in cancer patients [16]. Several well established methods of FP have been introduced, including embryo cryopreservation, gonadal shielding during radiotherapy, trachelectomy, and ovarian transposition (Table 2).

Embryo cryopreservation is a well established technique and the current live-birth rate per transfer using frozen thawed embryos was 35.6\% in US women under 35-year-old (http://www.sart.org, 2008). Embryo freezing should initially be considered for FP treatment if there is adequate time for ovarian stimulation and a partner or donor sperm.

Gonadal shielding during radiotherapy should be considered if radiotherapy is required for cancer treatment. For example, radiation plays an important role in the management of breast cancer, which can be classified into four categories: 1) Primary radiotherapy in breastconserving treatment for early breast cancer, 2) Adjuvant radiotherapy after mastectomy for high-risk patients, 3) Radiotherapy after neoadjuvant chemotherapy in locally-advanced breast cancers, and 4) 
Table 2. Fertility preservation options in females

\begin{tabular}{ll}
\hline Standard methods & \multicolumn{1}{c}{ Experimental methods } \\
\hline Embryo cryopreservation & Oocyte cryopreservation \\
Gonadal shielding during radiation therapy & Ovarian cryopreservation and transplantation \\
Ovarian transposition (oophoropexy) & Ovarian suppression with GnRH analogs or antagonists \\
Trachelectomy & \\
Other conservative gynecologic surgery & \\
\hline
\end{tabular}

Table 3. Indications for trachelectomy in cervical cancer

1. Women who desire to preserve fertility (age < 40-45)

2. Stage la ${ }^{\mathrm{a}}$ (with lymph vascular space involvement), la2, lb1

3. Lesion size $\leq 2 \mathrm{~cm}$

4. Histologically squamous, adeno-, or adenosquamous carcinoma

5. No upper cervical canal involvement of cancer

6. No evidence of lymph node metastasis

${ }^{a}$ Conization, la1 without lymph vascular space involvement.

Palliative radiotherapy for metastatic disease [17].

Trachelectomy is also an accepted method for the surgical management of early stage cervical cancer in women who wish to preserve their fertility. Cervical cancer is one of the most common cancers in women younger than 40 along with breast cancer, non-Hodgkin's lymphoma, and leukemia [18]. Indications for trachelectomy are described in Table 3.

Plante et al. [19] reported their experience with 125 patients who underwent vaginal radical trachelectomy. The recurrence rate of cervical cancer after trachelectomy was less than $5 \%$ and the death rate was less than $2 \%$. Having lesions sized $>2 \mathrm{~cm}$ was a strong risk factor for recurrence. A total of 58 women out of 125 conceived a total of 106 pregnancies, and of those, $73 \%$ of pregnancies reached the third trimester, of which, in turn, $75 \%$ gave birth at term. Overall, $13.5 \%$ of patients were associated with fertility problems.

Ovarian transposition can be performed not only for preservation of fertility but also for preventing premature ovarian failure in cervical cancer patients who will be treated with radiotherapy. It is necessary for gynecologic oncologists to understand the field of radiotherapy to prepare for ovarian transposition. Usually, standard fields are used with the upper field border on the fourth/fifth lumbar vertebra [20]. It is widely accepted that surgical transposition should be at least $3 \mathrm{~cm}$ above the upper border of the radiation field [21]. Hwang et al. [22] reported that a location for the transposed ovary higher than 1.5 $\mathrm{cm}$ above the iliac crest is recommended to avoid ovarian failure after primary or adjuvant pelvic radiotherapy in cervical cancer.

To date, the remaining methods are considered to be experimental, although the oocyte survival rate $(81 \%$ vs. $68 \%)$ and live-birth rate per embryo transfer (34\% vs. $14 \%$ ) of oocyte cryopreservation with vitrification is significantly higher than with conventional slow freez-
Table 4. Worldwide pregnancies from ovarian tissue cryopreservation and reimplantation [25]

\begin{tabular}{lccc}
\hline Case & Diagnosis & No. of babies & Main researcher \\
\hline 1 & Hodgkin's lymphoma & 1 & Donnez \\
2 & Neurotumor & 1 & Donnez \\
3 & Non-Hodgkin's lymphoma & 1 & Meirow \\
4 & Hodgkin's lymphoma & 1 & Demeestere \\
5 & Ewing's sarcoma & 3 & Andersen \\
6 & Hodgkin's lymphoma & 1 & Andersen \\
7 & Premature ovarian failure & 1 & Silber \\
8 & Hodgkin's lymphoma & 2 & Silber \\
9 & Polyangiitis & 1 & Piver \\
10 & Breast cancer & 2 & Pellicer \\
11 & Sickle cell anemia & 1 & Piver \\
12 & Hodgkin's lymphoma & 2 & Revel
\end{tabular}

A total of 12 patients

A total of 17 babies $A$ total of 8 centers

ing methods [23]. Noyes et al. [24] asked whether it is"time to remove the experimental label" of oocyte cryopreservation. Because of the improvements in advanced technologies including freezing-thawing methods, a new guideline is necessary to update FP specialists with the latest knowledge.

\section{Ovarian function after ovarian transplantation}

Ovarian tissue cryopreservation and reimplantation is a main option tfor preserving the fertility of cancer patients who need cancer treatments without delay or do not want to undergo ovarian stimulation. For prepubertal girls diagnosed with cancer, ovarian tissue freezing is the only option for FP. To date, a total of 17 babies from 12 patients have been born worldwide from ovarian tissue cryopreservation and reimplantation [25] (Table 4). Based on the site of reimplantation of cryopreserved ovarian tissue, transplantation can be classified into two different types: orthotopic and heterotopic transplantation.

In addition, transplantation of cryopreserved ovarian tissue has shown to be a potential method for recovery of ovarian function [26]. Advantages of ovarian tissue transplantation are not only preserving fertility but also restoring endocrine function in young women after cancer treatment. In a review of successful orthotopic frozen-thawed 
ovarian reimplantation, restoration of ovarian activity was shown between 3.5 and 6.5 months after transplantation [27]. Oktay et al. [26, 28] reported the first case of laparoscopic transplantation of frozenthawed ovarian tissue into the pelvic sidewall with subsequent ovulation and subcutaneous ovarian transplantation to the forearm, which resulted in preserved endocrine function and follicular development. Approximately 10 weeks after ovarian transplantation to the forearm, endocrine function was restored with decreased follicle-stimulating hormone and luteinizing hormone levels, and cyclical variation of peripheral estradiol levels [28]. Silber reported the recovery of ovarian function in terms of recovery of menstrual cycles and hormone levels (FSH) approximately 80 to 140 days after reimplantation from their fresh and frozen transplant cases [25].

\section{Breast cancer and ovarian stimulation with aromatase inhibitor}

Breast cancer is the most common cancer in women of reproductive age in the US [29]. Most women with breast cancer require adjuvant chemotherapy including cyclophosphamide. At an average age of 40, administration of cyclophosphamide, methotrexate, and CMF or adriamycin, cyclophosphamide and taxol $(\mathrm{AC}+\mathrm{T})$ resulted in amenorrhea in $20 \%$ to $100 \%$ or $37 \%$ to $77 \%$ of patients followed up 1 year after adjuvant chemotherapy, respectively [30].

For FP, ovarian stimulation with gonadotropins for embryo or oocyte cryopreservation results in excessive levels of estrogen production. To reduce estrogen exposure during ovarian stimulation in hormone-dependent cancer, a novel protocol using letrozole (a third generation aromatase inhibitor) and gonadotropins was developed [31]. Use of aromatase inhibitors is increasingly common in the treatment of breast cancer [32]. They have also recently been introduced for ovulation induction. When compared to clomiphene cycles, they produce comparable results with peak levels even lower than the natural cycle. Because of their dual effect, they can be used in breast and endometrial cancer patients for ovulation induction. Tamoxifen cannot be used in endometrial cancer as it is antagonistic to the endometrium. Based on our unpublished data, long-term follow-up for up to 7 years, the letrozole protocol showed safe, efficient, and ageappropriate pregnancy success rates.

Ovarian stimulation for embryo or oocyte cryopreservation should be started within the first four days of the menstrual cycle. Depending on whether the referral is made before or after breast surgery, usually no more than one or two cycles of ovarian stimulation can be performed without delaying chemotherapy in women with breast cancer [12]. This delay is generally acceptable as multiple studies have shown no effect on survival or recurrence in breast cancer patients if chemotherapy had been initiated as late as 12 weeks after breast sur- gery [33,34].

In FP cycles, since there is limited time available prior to the onset of chemotherapy in breast cancer patients [12], ovarian stimulation is often performed with higher doses of gonadotropins to maximize the number of embryos or oocytes cryopreserved, and to increase the likelihood of future pregnancies. We compared a low dose FSH start (=150 IU) with a high dose (>150 IU) in women with breast cancer undergoing FP with letrozole and found that the higher dose FSH stimulation in letrozole cycles did not improve pregnancy outcomes, and may be associated with a lower live birth rate [35]. In addition, it may increase estrogen exposure as well as the costs of FP.

\section{Ovarian suppression to prevent gonadal damage}

Given the current evidence, both the efficacy and safety of the use of GnRH agonists or antagonists during chemotherapy for prevention of gonadal damage are controversial. Several studies have suggested a decreased incidence of amenorrhea with the use of GnRH agonists throughout chemotherapy; however, most have been nonrandomized or small sample sized studies. Only five randomized trials have been completed.

Badawy et al. [36] and Del Mastro et al. [37] demonstrated the positive effect of $\mathrm{GnRH}$ agonists on the resumption of menses and ovulation; however, Leonard et al. [38], Gerber et al. [39], and Munster et al. [40] revealed that there was no impact of GnRH agonists on the prevention of early menopause or ovarian function.

Recently, Partridge [41] reported that women who are interested in future fertility and the providers who are assisting them should not depend on $\mathrm{GnRH}$ agonist treatment during chemotherapy for preservation of menstrual and ovarian function or fertility. There is one randomized ongoing study by the Southwest Oncology Group, called the Prevention of Early Menopause Study, a large international multicenter trial. This study will provide additional complementary information including biomarkers of ovarian reserve and rates of long-term amenorrhea or premature ovarian failure in women with or without ovarian suppression throughout treatment.

\section{Conclusion}

Recently, Tilly's team from the Vincent Center for Reproductive Biology at Massachusetts General Hospital isolated oocyte-producing stem cells from the ovaries of reproductive women and showed that these cells can produce normal oocytes. This study opened a new research field in human reproductive biology and may offer new opportunities to expand on and enhance current fertility-preservation strategies [42]. 
According to the development of early diagnosis and cancer treatments, techniques for FP including ART and freezing/thawing methods are continuously being developed. In addition, numerous experimental studies have been performed such as whole ovary transplantation and development of angiogenesis factors to avoid ischemic damage after ovarian tissue transplantation.

To provide better treatments for preserving fertility in women with cancer, physicians should recognize the determinants to access to FP and factors favoring referrals, and should make an effort to remove those barriers. Also, physicians who take part in the initial diagnosis of cancer should consider the importance of early referral of young cancer patients to FP specialists and take care of those patients by providing timely information and appropriate counseling. We believe that individualized treatment strategies should be delivered depending on the patient's situation with an appropriate team approach.

\section{Conflict of interest}

No potential conflict of interest relevant to this article was reported.

\section{References}

1. American Cancer Society. Breast cancer facts \& figures $2005-2006$ [Internet]. Atlanta: American Cancer Society Inc.; c2009 [2012 Jun 27]. Available from: http://www.cancer.org/acs/groups/content/@nho/documents/document/caff2005brfacspdf2005pdf. pdf.

2. Kovács P, Mátyás S, Ungár L. Preservation of fertility in reproductive-age women with the diagnosis of cancer. Eur J Gynaecol Oncol 2008;29:425-34.

3. Rodriguez-Wallberg KA, Oktay K. Options on fertility preservation in female cancer patients. Cancer Treat Rev 2012;38:354-61.

4. Sklar C. Maintenance of ovarian function and risk of premature menopause related to cancer treatment. J Natl Cancer Inst Monogr 2005:25-7.

5. Wallace WH, Thomson AB, Saran F, Kelsey TW. Predicting age of ovarian failure after radiation to a field that includes the ovaries. Int J Radiat Oncol Biol Phys 2005;62:738-44.

6. Wo JY, Viswanathan AN. Impact of radiotherapy on fertility, pregnancy, and neonatal outcomes in female cancer patients. Int J Radiat Oncol Biol Phys 2009;73:1304-12.

7. Green DM, Sklar CA, Boice JD Jr, Mulvihill JJ, Whitton JA, Stovall M, et al. Ovarian failure and reproductive outcomes after childhood cancer treatment: results from the Childhood Cancer Survivor Study. J Clin Oncol 2009;27:2374-81.

8. Chemaitilly W, Mertens AC, Mitby P, Whitton J, Stovall M, Yasui Y, et al. Acute ovarian failure in the childhood cancer survivor study.
J Clin Endocrinol Metab 2006;91:1723-8.

9. Oktem 0, Oktay K. Fertility preservation for breast cancer patients. Semin Reprod Med 2009;27:486-92.

10. Azim A, Rauch ER, Ravich M, Witkin S, Oktay K. Ovarian reserve is impaired in cancer patients with normal baseline FSH who previously received chemotherapy as determined by response to controlled ovarian stimulation and anti-mullerian hormone measurements: a controlled study. Fertil Steril 2006;86 (Suppl 2):S123-4.

11. Lee S, Heytens E, Moy F, Ozkavukcu S, Oktay K. Determinants of access to fertility preservation in women with breast cancer. Fertil Steril 2011;95:1932-6.

12. Lee S, Ozkavukcu S, Heytens E, Moy F, Oktay K. Value of early referral to fertility preservation in young women with breast cancer. J Clin Oncol 2010;28:4683-6.

13. Quinn GP, Vadaparampil ST, Lee JH, Jacobsen PB, Bepler G, Lancaster J, et al. Physician referral for fertility preservation in oncology patients: a national study of practice behaviors. J Clin Oncol 2009;27:5952-7.

14. Forman EJ, Anders CK, Behera MA. Pilot survey of oncologists regarding treatment-related infertility and fertility preservation in female cancer patients. J Reprod Med 2009;54:203-7.

15. Armuand GM, Rodriguez-Wallberg KA, Wettergren L, Ahlgren J, Enblad G, Hoglund $M$, et al. Sex differences in fertility-related information received by young adult cancer survivors. J Clin Oncol 2012;30:2147-53.

16. Lee SJ, Schover LR, Partridge AH, Patrizio P, Wallace WH, Hagerty $\mathrm{K}$, et al. American Society of Clinical Oncology recommendations on fertility preservation in cancer patients. J Clin Oncol 2006;24: 2917-31.

17. Suh CO. Radiotherapy for breast cancer. J Korean Med Assoc 2003; 46:503-11.

18. Jemal A, Murray T, Ward E, Samuels A, Tiwari RC, Ghafoor A, et al. Cancer statistics, 2005. CA Cancer J Clin 2005;55:10-30.

19. Plante M, Gregoire J, Renaud MC, Roy M. The vaginal radical trachelectomy: an update of a series of 125 cases and 106 pregnancies. Gynecol Oncol 2011;121:290-7.

20. Marnitz S, Köhler C, Schneider A, Seiler F, Hinkelbein W. Interindividual variability of lymph drainages in patients with cervical cancer. Implication on irradiation planning. Strahlenther Onkol 2006; 182:80-5.

21. Martin JR, Kodaman P, Oktay K, Taylor HS. Ovarian cryopreservation with transposition of a contralateral ovary: a combined approach for fertility preservation in women receiving pelvic radiation. Fertil Steril 2007;87:189.e5-7.

22. Hwang JH, Yoo HJ, Park SH, Lim MC, Seo SS, Kang S, et al. Association between the location of transposed ovary and ovarian function in patients with uterine cervical cancer treated with (post- 
operative or primary) pelvic radiotherapy. Fertil Steril 2012;97: 1387-93.e2.

23. Noyes N, Porcu E, Borini A. Over 900 oocyte cryopreservation babies born with no apparent increase in congenital anomalies. Reprod Biomed Online 2009;18:769-76.

24. Noyes N, Boldt J, Nagy ZP. Oocyte cryopreservation: is it time to remove its experimental label? J Assist Reprod Genet 2010;27: 69-74.

25. Silber SJ. Ovary cryopreservation and transplantation for fertility preservation. Mol Hum Reprod 2012;18:59-67.

26. Oktay K, Karlikaya G. Ovarian function after transplantation of frozen, banked autologous ovarian tissue. N Engl J Med 2000; 342:1919.

27. Donnez J, Silber S, Andersen CY, Demeestere I, Piver P, Meirow D, et al. Children born after autotransplantation of cryopreserved ovarian tissue. a review of 13 live births. Ann Med 2011;43:43750.

28. Oktay K, Economos K, Kan M, Rucinski J, Veeck L, Rosenwaks Z. Endocrine function and oocyte retrieval after autologous transplantation of ovarian cortical strips to the forearm. JAMA 2001; 286:1490-3.

29. American Cancer Society. Cancer facts \& figures 2009 [Internet]. Atlanta: American Cancer Society Inc.; c2009 [2012 Jun 27]. Available from: http://www.cancer.org/acs/groups/content/@nho/ documents/document/500809webpdf.pdf.

30. Sonmezer M, Oktay K. Fertility preservation in young women undergoing breast cancer therapy. Oncologist 2006;11:422-34.

31. Azim AA, Costantini-Ferrando M, Oktay K. Safety of fertility preservation by ovarian stimulation with letrozole and gonadotropins in patients with breast cancer: a prospective controlled study. J Clin Oncol 2008;26:2630-5.

32. Goss PE, Ingle JN, Martino S, Robert NJ, Muss HB, Piccart MJ, et al. A randomized trial of letrozole in postmenopausal women after five years of tamoxifen therapy for early-stage breast cancer. N Engl J Med 2003;349:1793-802.

33. Lohrisch C, Paltiel C, Gelmon K, Speers C, Taylor S, Barnett J, et al. Impact on survival of time from definitive surgery to initiation of adjuvant chemotherapy for early-stage breast cancer. J Clin On- col 2006;24:4888-94.

34. Cold S, Düring M, Ewertz M, Knoop A, Møller S. Does timing of adjuvant chemotherapy influence the prognosis after early breast cancer? Results of the Danish Breast Cancer Cooperative Group (DBCG). Br J Cancer 2005;93:627-32.

35. Lee S, Oktay K. Does higher starting dose of FSH stimulation with letrozole improve fertility preservation outcomes in women with breast cancer. Fertil Steril 2010;94 Suppl:S160.

36. Badawy A, Elnashar A, El-Ashry M, Shahat M. Gonadotropin-releasing hormone agonists for prevention of chemotherapy-induced ovarian damage: prospective randomized study. Fertil Steril 2009;91:694-7.

37. Del Mastro L, Boni L, Michelotti A, Gamucci T, Olmeo N, Gori S, et al. Effect of the gonadotropin-releasing hormone analogue triptorelin on the occurrence of chemotherapy-induced early menopause in premenopausal women with breast cancer: a randomized trial. JAMA 2011;306:269-76.

38. Leonard RC, Adamson D, Anderson R, Ballinger R, Bertelli G, Coeman RE, et al. The OPTION trial of adjuvant ovarian protection by goserelin in adjuvant chemotherapy for early breast cancer [abstract]. J Clin Oncol 2010;28 (Suppl 15):89s.

39. Gerber B, von Minckwitz G, Stehle H, Reimer T, Felberbaum R, Maass $\mathrm{N}$, et al. Effect of luteinizing hormone-releasing hormone agonist on ovarian function after modern adjuvant breast cancer chemotherapy: the GBG 37 ZORO study. J Clin Oncol 2011; 29:2334-41.

40. Munster PN, Moore AP, Ismail-Khan R, Cox CE, Lacevic M, GrossKing $M$, et al. Randomized trial using gonadotropin-releasing hormone agonist triptorelin for the preservation of ovarian function during (neo) adjuvant chemotherapy for breast cancer. J Clin Oncol 2012;30:533-8.

41. Partridge AH. Ovarian suppression for prevention of premature menopause and infertility: empty promise or effective therapy? J Clin Oncol 2012;30:479-81.

42. White YA, Woods DC, Takai Y, Ishihara O, Seki H, Tilly JL. Oocyte formation by mitotically active germ cells purified from ovaries of reproductive-age women. Nat Med 2012;18:413-21. 